\title{
Performance of high-resolution X-band weather radar networks - the PATTERN example
}

\author{
K. Lengfeld ${ }^{1}$, M. Clemens ${ }^{1}$, H. Münster ${ }^{2}$, and F. Ament ${ }^{1,2}$ \\ ${ }^{1}$ Meteorological Institute of the University of Hamburg, Hamburg, Germany \\ ${ }^{2}$ Max-Planck-Institute for Meteorology, Hamburg, Germany \\ Correspondence to: K. Lengfeld (katharina.lengfeld@zmaw.de)
}

Received: 25 July - Published in Atmos. Meas. Tech. Discuss.: 8 August 2014

Revised: 31 October 2014 - Accepted: 31 October 2014 - Published: 5 December 2014

\begin{abstract}
This publication intends to prove that a network of low-cost local area weather radars (LAWR) is a reliable and scientifically valuable complement to nationwide radar networks. A network of four LAWRs has been installed in northern Germany within the framework of the Precipitation and Attenuation Estimates from a High-Resolution Weather Radar Network (PATTERN) project observing precipitation with a temporal resolution of $30 \mathrm{~s}$, a range resolution of $60 \mathrm{~m}$ and a sampling resolution of $1^{\circ}$ in the azimuthal direction. The network covers an area of $60 \mathrm{~km} \times 80 \mathrm{~km}$. In this paper, algorithms used to obtain undisturbed precipitation fields from raw reflectivity data are described, and their performance is analysed. In order to correct operationally for background noise in reflectivity measurements, noise level estimates from the measured reflectivity field are combined with noise levels from the last 10 time steps. For detection of non-meteorological echoes, two different kinds of clutter algorithms are applied: single-radar algorithms and networkbased algorithms. Besides well-established algorithms based on the texture of the logarithmic reflectivity field (TDBZ) or sign changes in the reflectivity gradient (SPIN), the advantage of the unique features of the high temporal and spatial resolution of the network is used for clutter detection. Overall, the network-based clutter algorithm works best with a detection rate of up to $70 \%$, followed by the classic TDBZ filter using the texture of the logarithmic reflectivity field.

A comparison of a reflectivity field from the PATTERN network with the product from a C-band radar operated by the German Meteorological Service indicates high spatial accordance of both systems in the geographical position of the rain event as well as reflectivity maxima. Long-term statistics from May to September 2013 prove very good accordance of
\end{abstract}

the X-band radar of the network with C-band radar, but, especially at the border of precipitation events, higher-resolved $\mathrm{X}$-band radar measurements provide more detailed information on precipitation structure because the $1 \mathrm{~km}$ range gate of $\mathrm{C}$-band radars is only partially covered with rain. The standard deviation within a range gate of the C-band radar with a range resolution of $1 \mathrm{~km}$ is up to $3 \mathrm{dBZ}$ at the borders of rain events. The probability of detection is at least $90 \%$, the false alarm ratio less than $10 \%$ for both systems. Therefore, a network of high-resolution low-cost LAWRs can give valuable information on the small-scale structure of rain events in areas of special interest, e.g. urban regions, in addition to the nationwide radar networks.

\section{Introduction}

Flood forecasting, urban hydrology, hydrometeorological applications and management of risk and uncertainty require high-resolution spatial and temporal rainfall estimates near the ground with less than $0.1 \mathrm{~km}$ and $1 \mathrm{~min}$, respectively (Einfalt, 2003). Therefore, radar systems capable of producing reliable and accurate quantitative estimates of precipitation at high temporal and spatial resolution are needed. Rainfall products of conventional radar systems used in nationwide or even larger networks are generally based on reflectivity measurements at $\mathrm{S}$ - or C-band frequencies with a temporal resolution of several minutes and a spatial resolution of a few hundred metres.

To meet present and future demands of resolution, radar observations at shorter wavelengths are a promising option, as the resolution depends, among other factors, on the width 
of the antenna and the wavelength. Therefore, recent studies support observations at $\mathrm{X}$-band frequencies as an alternative or an addition to S- and C-bands (Lengfeld et al., 2013; Trabal et al., 2013) to fulfil the requirements of urban drainage system modelling as input for rainfall-runoff models of rural river systems, hydraulic simulations, insurance proof, detailed information on extreme events, and many more. Besides higher resolution, radars operating at high frequencies benefit from lower costs resulting from smaller antenna size compared to long-wave radars. X-band radars can also derive reliable precipitation estimates close to the ground due to their relatively short range. S- or C-band radars measure within a range of hundreds of kilometres. Therefore, they cannot observe rainfall near the ground, because the radar beam increases in height with increasing distance to the radar due to the elevation angle and the Earth's curvature. Measurements taken at a few kilometres in height above the Earth's surface need to be extrapolated to give an estimation of rainfall on the ground. These techniques are limited and imprecise, leading to large uncertainties in estimated reflectivity.

In contrast to long wavelengths, reflectivity measurements at shorter wavelengths, especially at X-and K-bands, are significantly attenuated by liquid water along their paths, where the specific attenuation at any distance depends on the size distribution of raindrops and their extinction cross section. The magnitude of attenuation is generally inverse to the wavelength, and the specific attenuation at the X-band is approximately 2 orders of magnitude higher than at the S-band, according to Doviak and Zrnic (1993). While the effect of attenuation at the S-band is practically negligible, it becomes increasingly serious as the wavelength is reduced, and corrections have to be applied to retrieve intrinsic reflectivity. The basics of microwave attenuation by rain have already been discussed by many authors, e.g. Atlas and Banks (1951), Hitschfeld and Bordan (1954), Gunn and East (1954), Wexler and Atlas (1963), Dutton (1967) and Atlas and Ulbrich (1977), to mention the earliest.

To overcome the apparent drawback of strong attenuation at X-band frequencies, several authors introduced concepts of overlapping radar networks consisting of two or more radars. These methods allow for estimation of attenuation and correction of observed reflectivities simultaneously, if at least two radars observe a common volume (Testud and Amayenc, 1989; Kabeche and Testud, 1995; Srivastava and Tian, 1996; Tian and Srivastava, 1997; Chandrasekar and Lim, 2008; Lim et al., 2011). In the last few years, several networks consisting of different types of X-band radars have been installed to fill gaps in nationwide networks of C- or S-band radars, e.g. within the CASA (Collaborative Adaptive Sensing of the Atmosphere) project (Trabal et al., 2013; Mahale et al., 2014), in complex terrain, or in areas where detailed information on rainfall is of high interest, e.g. near airports (Turso et al., 2009), in mountainous regions (Beck and Bousquet, 2013; Shakti et al., 2013; Ventura and Tabary,
2013) or in flood-prone areas (Matrosov et al., 2013), to name a few of them.

Comparing the existing X-band radar networks, one can distinguish between three different approaches. On the one hand, there are highly sensitive X-band weather radar systems featuring dual polarisation as a standard, with a peak power of about $75 \mathrm{~kW}$ and a typical antenna size of $2.5 \mathrm{~m}$ in diameter (e.g. Beck and Bousquet, 2013). The maximum range is of the order of $100 \mathrm{~km}$, with a range resolution of $20 \mathrm{~m}$. On the other hand, there is an increasing number of low-cost systems mainly based on conventional nautical radar systems with a peak power of $25 \mathrm{~kW}$ (e.g. Trabal et al., 2013; Lengfeld et al., 2012). These systems are characterised by small antenna diameters of less than $1 \mathrm{~m}$ and, in general, they are not capable of performing measurements in Doppler or dual-polarisation mode. The maximum range of these low-cost radars is of the order of $20 \mathrm{~km}$, with a range resolution similar to the former X-band radar type. Due to their limited range, they are often called local area weather radars (LAWR). These systems can serve as a sort of magnifying glass in complementation to C- or S-band radar systems in flood-prone regions or other areas of interest, because they are affordable not only for weather services, but also for local authorities or private companies (10-20\% of the price of dual-polarisation X-band radars). A third approach is systems with a low peak power of $25 \mathrm{~kW}$ that perform dualpolarisation measurements with a maximum range of the order of $100 \mathrm{~km}$ and a range resolution of the order of $250 \mathrm{~m}$ (e.g. Barbieri et al., 2014).

A network of four LAWRs is installed in northern Germany within the framework of the Precipitation and Attenuation Estimates from a High-Resolution Weather Radar Network (PATTERN) project. The goal is to demonstrate that low-cost radar systems are a scientifically valuable tool for investigating the spatial structure of precipitation and that a network of LAWRs can enhance the quality of the retrieved precipitation field. The network approach based on high-resolution X-band radars has two definite advantages compared to large-scale C-band radar networks: gain of additional information arises from high resolution (temporal and spatial) as well as from the wide overlapping areas of multiple coverage. The former results from the technical specifications of the X-band radar itself, e.g. wavelength, rotational speed of the antenna, pulse length and repetition frequency. The latter is based on the network set-up that is not designed to cover an area as large as possible like nationwide C-band radar networks, but to create large overlapping areas covered by multiple radars. Overall, these advantages could not only lead to better attenuation estimates, but also to improvements in several retrievals regarding clutter detection, gap filling and, finally, the estimation of precipitation. Therefore, we will provide high-quality precipitation data in high temporal and spatial resolution that could be beneficial for rainfall-runoff simulation, for example. In addition, the Xband radars can serve as gap fillers and provide precipitation 


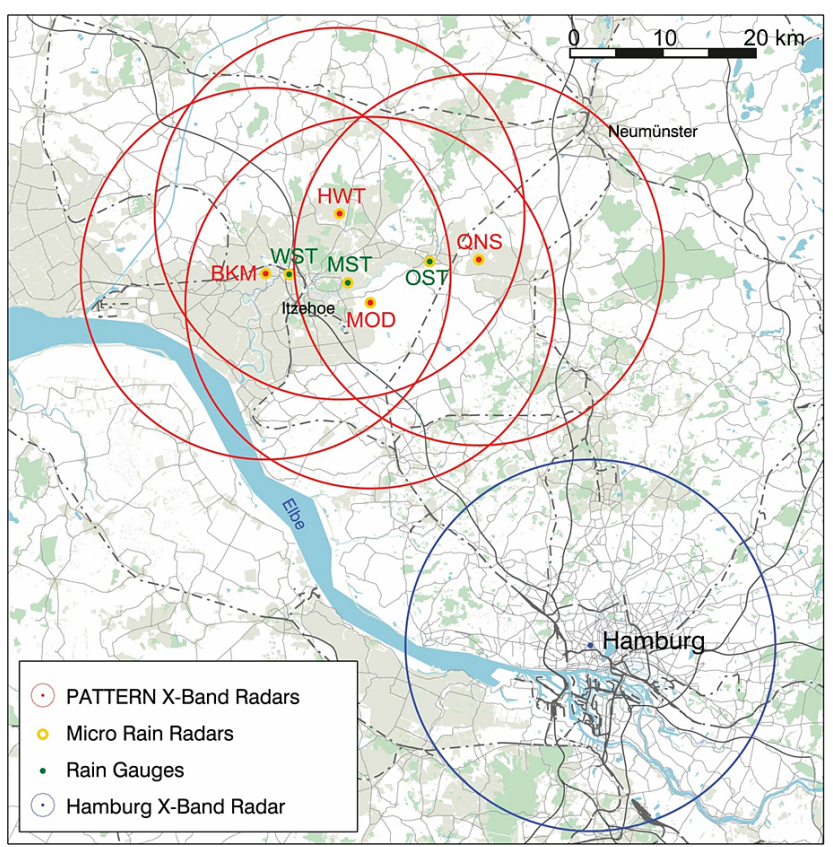

Figure 1. Position of the four network radars: Hungriger Wolf tower (HWT), Quarnstedt (QNS), Bekmünde (BKM) and Moordorf (MOD) marked in red, the Hamburg radar marked in blue, and their $20 \mathrm{~km}$ range. Reference stations OST, MST and WST are marked in green.

estimates near the ground (McLaughlin et al., 2009) in areas far away from C- or S-band radar sites where the radar beam is several hundred metres above the ground.

The aim of this paper is to identify advantages and disadvantages of the network as well as single $\mathrm{X}$-band radars and present algorithms making use of the network approach and the X-band-specific characteristics. The X-band radars used in this study are described in Sect. 2. It also gives an overview of the design of the network. Section 3 presents the algorithms that are applied to raw reflectivity data in order to get reliable reflectivity and precipitation fields. First, algorithms are described that are applied to individual radars, followed by algorithms that use the advantage of having overlapping areas within the network. A comparison with a large-scale Cband radar operated by the German Meteorological Service (DWD) in Sect. 4 gives a first evaluation of the quality of precipitation fields derived from the PATTERN network. In Sect. 5, conclusions are drawn, and Sect. 6 gives an outlook on future work.

\section{Radar network}

A network of four X-band radars, Hungriger Wolf tower (HWT), Quarnstedt (QNS), Bekmünde (BKM) and Moordorf (MOD), has been set up to the north of Hamburg, Germany (Fig. 1, Lengfeld et al., 2012), within the framework of the PATTERN project. Each radar has a range of $20 \mathrm{~km}$ in ra-

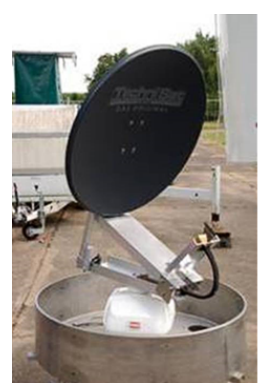

(a)

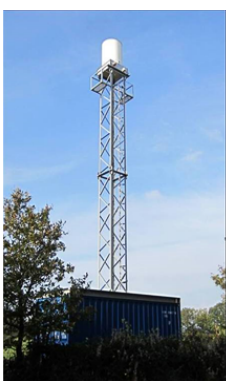

(b)

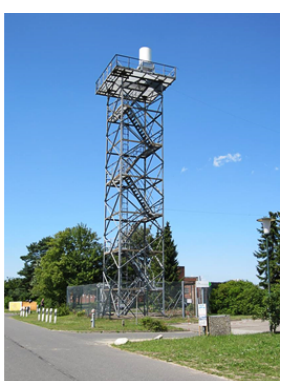

(c)
Figure 2. Modified ship navigation radar with parabolic dish (a), typical radar tower in Quarnstedt (b) and radar at Hungriger Wolf (c).

dius around the site to detect reflectivity. The network spans a region of approximately $60 \mathrm{~km} \times 80 \mathrm{~km}$. Two radars are at least $11 \mathrm{~km}$, but not more than $16 \mathrm{~km}$ apart. Based on the network design, a large area is covered by at least two radars at the border and up to four radars in the centre of the network. Therefore, multiple information from different radars is available on reflectivity and attenuation in a certain grid cell. This advantage can be used for clutter detection, gap filling and attenuation estimation. Additionally, micro rain radars (MRR) at each radar site complement the network. These vertically profiling K-band radars measure Doppler spectra of hydrometeors at 31 height levels. From these spectra, drop size distributions at each height level and, finally, reflectivity as well as rain-rate profiles are derived (Peters et al., 2005). To calibrate the X-band radars and to evaluate retrieved products, three reference stations (OST, MST and WST in Fig. 1) are set up within the PATTERN area, consisting of MRR and rain gauges corrected for wind speed at each site. The whole PATTERN region is covered by a Cband radar operated by the DWD.

The LAWRs used in PATTERN are modified ship navigation radars of type GEM scanner SU70-25E with $25 \mathrm{~kW}$ transmit power. Corresponding to technical specifications, the modified radars are simple backscatter systems and cannot observe Doppler shift, nor do they perform polarimetric measurements. The original fan beam antenna is replaced by a high-gain pencil beam antenna; in order to reduce side lobes, an offset parabolic dish of $0.85 \mathrm{~m}$ in diameter is used (Fig. 2a). Antenna and scanning drive are protected by a low-loss radome with air conditioning to avoid condensation within the radome. Due to the cylindrical shape of the radome, water runs off quickly in the case of a rain event, and attenuation due to a wet radome is minimised. Radar control, signal processing and data management is PC based. Technical details on radar systems and scanning schemes are listed in Table 1.

In the PATTERN set-up, LAWRs measure with a pulse width of $0.4 \mu \mathrm{s}$ at a pulse repetition frequency of $800 \mathrm{~Hz}$. 
Table 1. LAWR specifications.

\begin{tabular}{ll}
\hline Performance parameters & Specifications \\
\hline Range resolution & $60 \mathrm{~m}$ \\
Time resolution & $30 \mathrm{~s}$ \\
Angular resolution & $28^{\circ}$ \\
Sampling resolution in azimuth & $1^{\circ}$ \\
Maximum range & $20 \mathrm{~km}$ \\
Calibration accuracy & $\pm 1 \mathrm{~dB}$ \\
Transmit power & $25 \mathrm{~kW}$ \\
Frequency & $9410 \mathrm{MHz}$ \\
Pulse width & $0.4 \mu \mathrm{s}$ \\
Pulse repetition frequency & $800 \mathrm{~Hz}$ \\
Beam width & $2.8^{\circ}$ \\
\hline
\end{tabular}

Repetition frequency as well as continuous rotation with a speed of about $24 \mathrm{rpm}$ allow for reflectivity measurements with a temporal resolution of $30 \mathrm{~s}$. The beam width of the radar is $2.8^{\circ}$. Received reflectivities are averaged over a sequence of transmitted pulses with a sampling resolution of $1^{\circ}$ in the azimuthal direction. Due to the pulse repetition frequency of $800 \mathrm{~Hz}$ and continuous rotation of the antenna, the average is based on about 5 to 6 pulses per sweep, corresponding to 67 pulses per $1^{\circ}$ azimuthal range and an averaging interval of $30 \mathrm{~s}$. In comparison, the C-band radar operated by DWD derives reflectivities from only one sweep, with approximately 40 pulses per $1^{\circ}$ azimuth within about $50 \mathrm{~ms}$ of the $5 \mathrm{~min}$ interval the measurement is valid for. Therefore, the X-band radar benefits from a temporal average over the measuring period, in contrast to a snapshot within the measuring period as is derived by the $\mathrm{C}$-band radar. The scanning scheme of the X-band radar is azimuthal only, but fixed elevation angles can be adjusted for optimum operation according to site conditions. For the X-band radars of the PATTERN network, a fixed elevation of approximately $2^{\circ}$ is used.

The standard system installed at the sites consists of the radar mounted on a two-piece steel tower of $10 \mathrm{~m}$ in height screwed onto a steel frame on top of a container (Fig. 2b). The complete structure is $16 \mathrm{~m}$ high. In demounted state, the station fits into the container, and it can be moved easily. With the exception of HWT, all PATTERN radars are built using this standard installation. Radar HWT is placed on an already existing radar tower at the Hungriger Wolf former military airport (Fig. 2c).

Radars operating in the X-band frequency range benefit from lower costs resulting from smaller antenna size compared to long-wave radars. The systems used in this study are simple single-polarised systems that scan horizontally and do not observe Doppler shift. Therefore, they are inexpensive compared to dual-polarised Doppler X-band radars. A radar as it is used in this network costs approximately EUR 60000 including the data acquisition system and tower construction. This is less than $20 \%$ of conventional X-band radars. The network in this study includes four X-band radars, seven
MRRs and seven rain gauges. It was designed for research purposes and consists of more instruments than necessary for operational precipitation estimation in local areas. In operational use, it is possible to apply common adjustment procedures using ground-based precipitation observations by rain gauges or disdrometers. However, a vertical profiling instrument such as an MRR provides the opportunity to compare directly observed reflectivity within a common volume. One additional MRR and rain gauge in the area covered by all $\mathrm{X}$-band radars of a network would be sufficient to obtain reliable precipitation data. The overall price of the network depends on the kind of application and the area that should be observed. In complementation to larger-scale radars, e.g. C- or S-band radars, one or two X-band radars can be sufficient. Nevertheless, the network-based algorithms presented in this study are designed for networks of at least three Xband radars.

\section{Data processing}

Weather radars cannot measure precipitation directly; they measure reflectivity from particles along the radar path. Therefore, a raw reflectivity signal not only contains meteorological echoes from precipitation, but also nonmeteorological echoes (clutter) and background noise. Before reflectivities are recorded as $30 \mathrm{~s}$ averages, disturbances caused by other radars and radio links are effectively eliminated by filtering peaks within adjacent pulses: data of a single pulse at a specific range gate are omitted if it is $2.5 \mathrm{dBZ}$ larger than both the corresponding measurements at the previous and following pulses. This filter suppresses effectively interferences from other X-band sources. The filter is insensitive with respect to the selection of the threshold, since the disturbing signals are in general very strong. The value of $2.5 \mathrm{dBZ}$ was determined by analysing raw pulse-to-pulse radar data output and manual detection of artificial signals.

In the following, algorithms for single radars are presented for estimating background noise, calibrating the Xband radar network with MRR measurements and correcting for attenuation. Additionally, retrievals are described to identify non-meteorological echoes using single radar measurements as well as the advantage of having areas of multiple coverage within the network. The functionality of the algorithms is demonstrated exemplarily for a rain event on 15 May 2013 at 15:28:30 UTC observed by radar MOD. The raw reflectivity field of this event as measured by radar MOD also includes, besides precipitation, background noise, interferences and clutter (Fig. 3a). The rain event covers the western part of the radar image, with reflectivities of up to $50 \mathrm{dBZ}$, and stands out clearly from the background noise. Clutter is mainly evident in the centre close to the radar site as small-scale disturbances with high reflectivity values. 


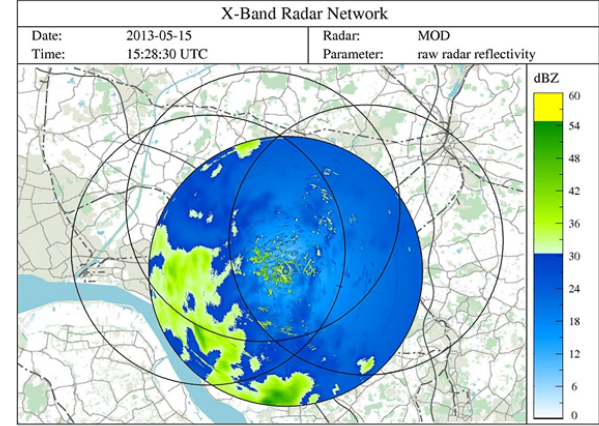

(a)

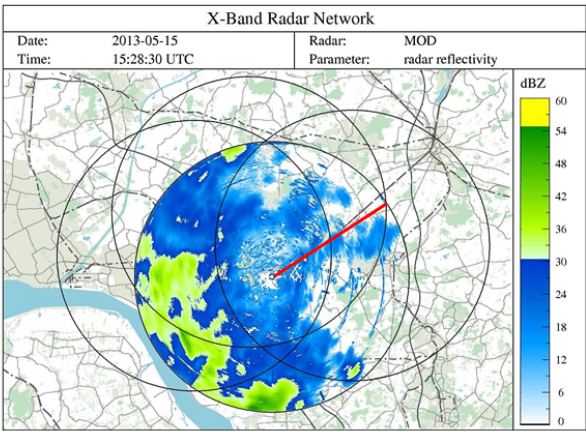

(b)

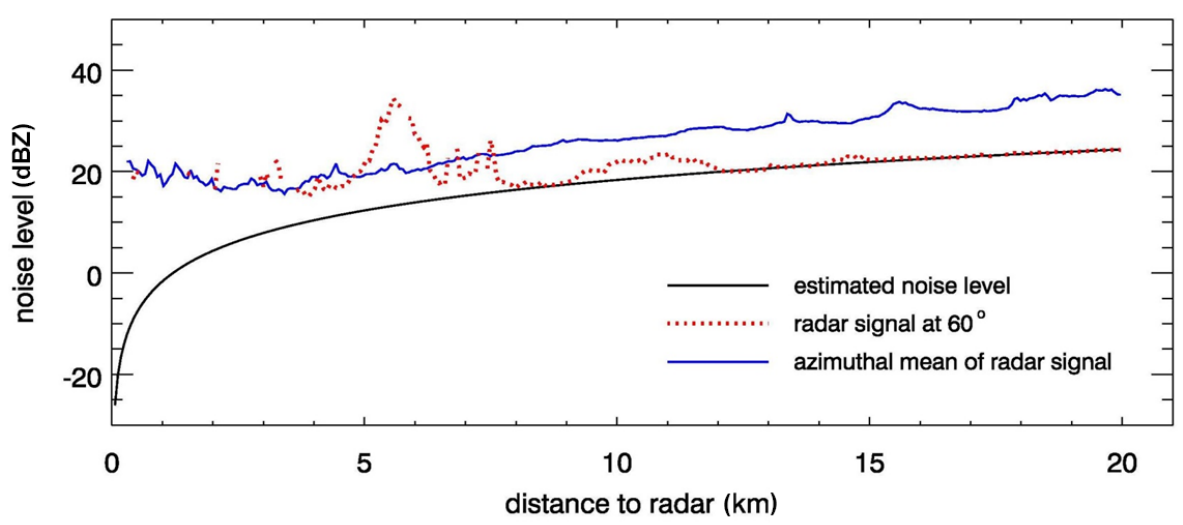

(c)

Figure 3. Estimating noise level for radar MOD for 15 May 2013, 15:28:30 UTC: (a) raw reflectivity field, (b) reflectivity field without background noise, with the red line indicating a $60^{\circ}$ azimuth, and (c) noise level and radar signal as a function of the distance to the radar.

\subsection{Noise detection}

All reflectivity measurements by radars are affected by noise from internal electrical circuits used in the receiver chain or by atmospheric noise from outside the system. An accurate estimation of the background noise is necessary, especially in the detection of weak weather signals. A detection algorithm based on received signals has to be implemented in the data processing, because the used LAWRs cannot measure the noise level directly. Power $P$ at the output of the GEM scanner SU70-25E system consists mainly of received power $P_{\mathrm{r}}$ due to weather signals and noise power $P_{\mathrm{N}}$. Received power $P_{\mathrm{r}}$ for distributed weather targets can be expressed as a function dependent on the radar system and physical parameters summarised by the weather radar constant $C$ as

$$
P_{\mathrm{r}}=\frac{C \cdot Z}{r^{2}}
$$

which is directly proportional to radar reflectivity factor $Z$ and inversely proportional to the square of distance $r$ (Skolnik, 2008). In contrast, the power of background noise $P_{\mathrm{N}}$ is independent of the distance to the radar. In a first step, an initial guess of the range-independent noise level from a rain-free field is used to separate assumed meteorological signals from noise background. This step is performed only in the very first time step after system start. If the initial guess is set too low and underestimates the actual noise level, some noise will remain in the radar image after subtracting the estimated background noise. Therefore, the initial first guess overestimates the expected noise level by approximately a factor of 10 . In case of more than $10 \%$ rainfree radar range gates after subtracting the initial guess from the original reflectivity field, the 10 th percentile of the original reflectivity field is chosen as the next noise level. Otherwise, the noise level from the last time step is kept. This estimated noise level is applied as the initial guess for noise estimation in the next $30 \mathrm{~s}$ time step. In order to minimise the influence of radar artefacts on the algorithm, the average of the recent 10 estimates is used to correct the measured reflectivity field for background noise. The estimated noise level is subtracted from each reflectivity field, which is then multiplied by the squared distance to the radar. The rangedependent received reflectivity $Z$ field due to weather signals and non-meteorological echoes remain (Fig. 3b). Noise level as a function of distance to the radar (black line in Fig. 3c) fits the radar signal at $60^{\circ}$ (red line in Fig. $3 \mathrm{~b}$ and red dotted line in Fig. 3c) for rain-free areas. At ranges with precipitation, the signal is the sum of noise and meteorological signal. Therefore, considering the rain field in Fig. 3b, the azimuthal 

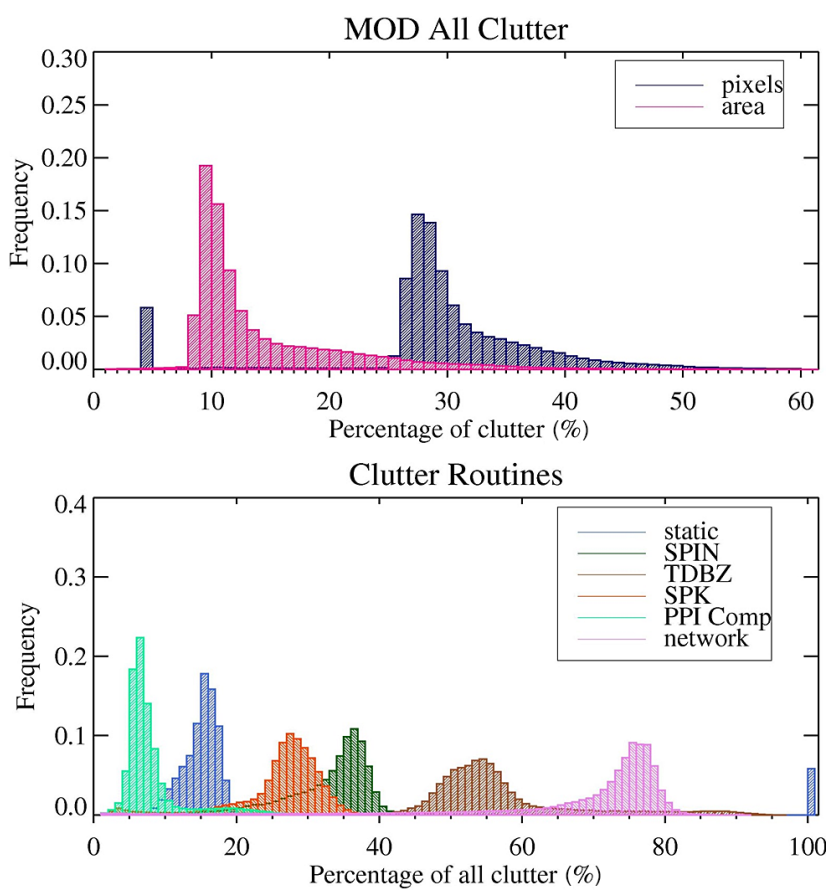

Figure 4. Distribution of clutter for the period from July to October 2013. (a) Histogram of the percentage of all clutter range gates (blue) and the clutter area (magenta). (b) Clutter detected by each algorithm (static clutter map in blue, SPIN filter in green, TDBZ filter in brown, spikes and ring algorithm in orange, time-resolutionbased algorithm in blue-green and network-based algorithm in violet) in percentage of all clutter range gates.

mean of the radar signal (blue line in Fig. 3c) is always higher than the estimated noise level.

\subsection{Clutter detection algorithms}

Advanced state-of-the-art methods to identify clutter are based on polarimetric measurements or observations of Doppler shift. The low-cost radars used in this study do not observe these quantities. Therefore, clutter detection is only based on temporal and spatial variability of reflectivity.

Clutter is characterised by high reflectivity values and can be divided into two different types: static and dynamic clutter. Static clutter is caused e.g. by trees, houses and natural reliefs, and is present in almost every data set at the same radar range gates. Dynamic clutter is caused by moving objects, e.g. birds and insects, or by other radars operating in the same frequency range (so-called interferences). It varies from time step to time step and from range gate to range gate. A number of correction algorithms are applied to reflectivity data of the PATTERN network in order to detect and delete clutter and interferences, so that the precipitation signal remains. Each correction algorithm pursues a different approach: some are based on common clutter identification methods for single radars, others use the unique features of high temporal resolution and large overlap of multiple radars within the network. Many clutter range gates fulfil detection criteria of more than one of these algorithms and are detected by multiple algorithms. Features of other clutter range gates can be detected by only one of the algorithms. In the following, these algorithms are described in more detail using the exemplary reflectivity field (Fig. 3b), and the performance of each algorithm will be investigated over a 4 month period.

The PATTERN network radar MOD is used exemplarily to investigate the performance of the clutter detection algorithms from July to October 2013. Around $30 \%$ of the radar range gates in each radar are classified as clutter by combining all detection algorithms (Fig. 4a). Most of the clutter range gates occur close to the radar. Therefore, about $10 \%$ of the area in the radar image is affected. The distribution is skewed to the right. This is due to a few disturbances that cannot be identified by the different clutter and interference detection algorithms, e.g. wide spikes. In Fig. 4b, each algorithm is considered separately. Most clutter range gates are identified by multiple algorithms. Therefore, the sum of all algorithms is higher than $100 \%$. The distribution of each clutter detection algorithm will be discussed in the following sections. The blue bar at $5 \%$ in Fig. $4 \mathrm{a}$ and at $100 \%$ in Fig. $4 \mathrm{~b}$ corresponds to cases where there were no radar measurements and the reflectivity field is empty. In these cases, no clutter detection algorithm is operational but the static clutter algorithm. Therefore, all clutter (100\%) identified in these cases is static clutter, which corresponds to approximately $5 \%$ of all range gates.

All range gates detected as clutter or interference are thresholded and removed after applying all algorithms. Two different approaches were used to fill these gates with information:

a. For individual radars, data gaps are filled by using an inverse distance weighting interpolation procedure with an area of influence of 50 range bins.

b. In regions covered by multiple radars within the network, gaps caused by clutter are filled using the information of at least one other radar or the averaged information where more than one radar is available.

\subsubsection{Static clutter algorithm}

The clutter easiest to identify is static clutter, because it is evident in almost every radar picture at the same range gates. To detect this type of clutter, a map is generated by counting the time steps at which reflectivity is higher than $7 \mathrm{dBZ}$ over 10 days, corresponding to 28800 time steps (Fig. 5a). That also includes the precipitation signal and dynamic clutter. The threshold is set to $7 \mathrm{dBZ}$, because clutter is characterised by reflectivity values clearly higher than the background noise, and to ensure that in the unlikely case of underestimation of background noise, clutter and the remaining background noise can be distinguished. A distinction 


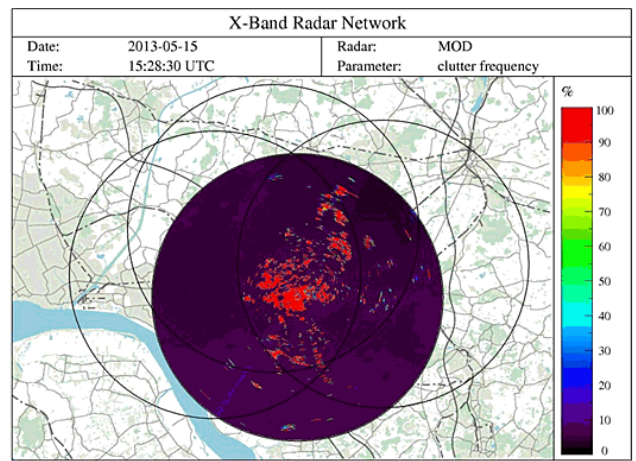

(a)

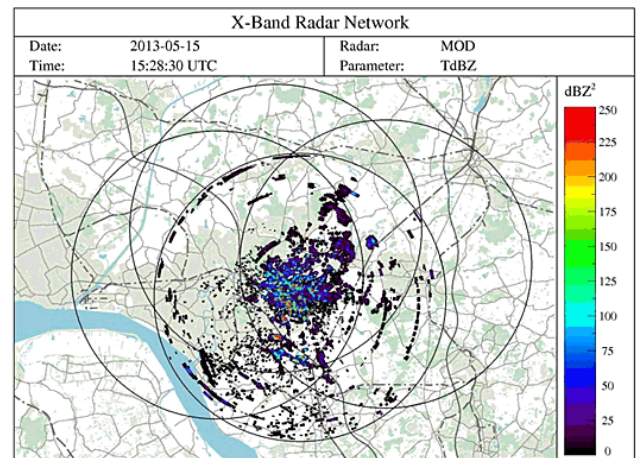

(c)

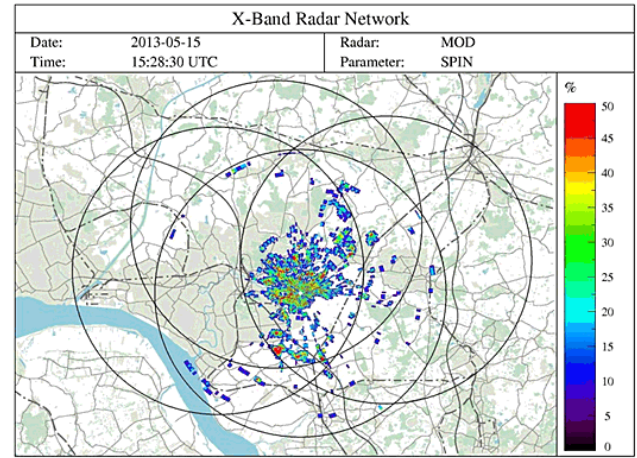

(e)

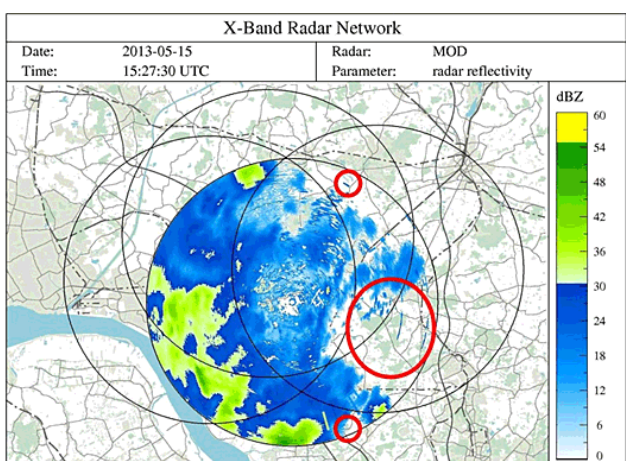

(b)

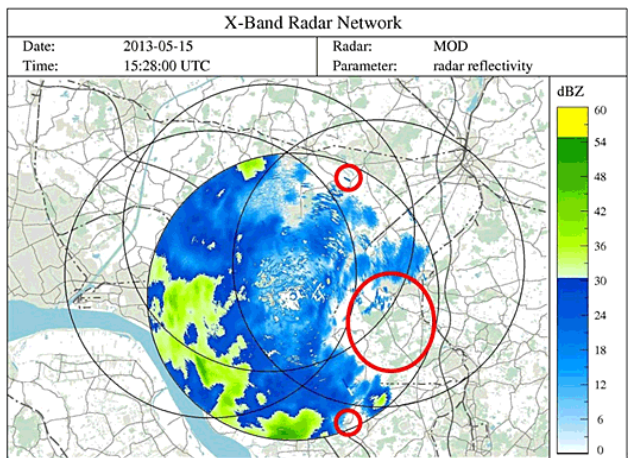

(d)

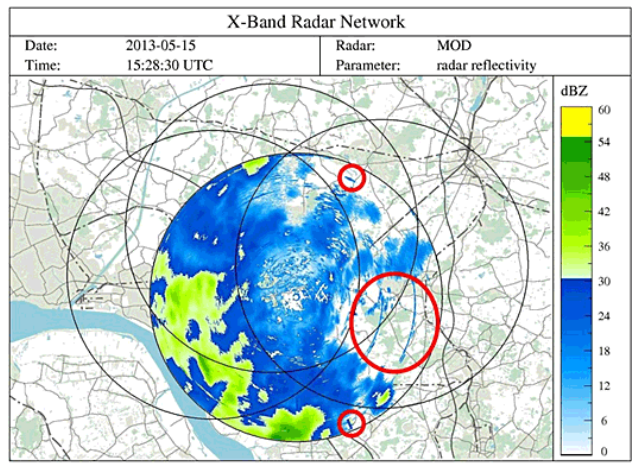

(f)

Figure 5. Clutter detection for radar MOD for 15 May 2013, 15:28:30 UTC: (a) static clutter map, (b) TDBZ field, (c) SPIN field. Clutter detection using previous PPIs: (d) dBZ field for 15:27:30 UTC, (e) dBZ field for 15:28:00 UTC, (f) dBZ field for 15:28:30 UTC.

between static clutter and long-lasting rainfall needs to be made. We assume at least $5 \%$ of the time period of ten days is rain free. Therefore, range gates that exceed the threshold in more than $95 \%$ of the 28800 time steps are marked as static clutter. 14-27\% of the range gates are identified as static clutter for the four network radars. That corresponds to $8-12 \%$ of the area covered, because static clutter primarily occurs in the vicinity of the radar where the radar beam is close to the ground.

The long-term study reveals that for radar MOD, up to $20 \%$ of all clutter range gates is static clutter (Fig. 4b). This means that around $80 \%$ of the clutter range gates are not static, but are dynamic clutter, and cannot be detected by the clutter map. Therefore, dynamic clutter algorithms need to be applied. In some images, $100 \%$ seems to be static clutter. This occurs if there are no measurements for certain time steps and if the other clutter detection algorithms do not operate.

\subsubsection{Dynamic clutter algorithms for single radars}

To identify dynamic clutter and interferences, several algorithms are applied based on the structure of the reflectivity 
field and a comparison to the last time steps. First, two common methods for detecting dynamic clutter are used: the texture of the logarithmic reflectivity (TDBZ) field, and the SPIN field (Hubbert et al., 2009). The TDBZ field is computed as the average of the squared logarithmic reflectivity difference between adjacent range gates:

$\mathrm{TDBZ}=\left[\sum_{i}^{N}\left(\mathrm{dBZ}_{i}-\mathrm{dBZ}_{i-1}\right)^{2}\right] / N$

where "dBZ" is reflectivity and $N$ is the number of range gates used. If the mean of squared reflectivity difference (Fig. 5b) within five consecutive range gates is higher than $3 \mathrm{dBZ}$, the range gate is flagged as clutter. If the threshold is set too high, some clutter remains undetected; if it is set too low, small-scale light rain events might falsely be classified as clutter. The threshold of $3 \mathrm{dBZ}$ is based on several case studies to optimise the performance of the algorithm. In order to optimise computing time, the TDBZ field is only calculated in the beam direction following Hubbert et al. (2009), and the number of consecutive range gates is limited to five.

The SPIN field (Fig. 5c) is a measure of how often the reflectivity gradient changes sign along the radial direction. Two conditions must be fulfilled:

$\operatorname{sign}\left\{X_{i}-X_{i-1}\right\}=-\operatorname{sign}\left\{X_{i+1}-X_{i}\right\}$

and

$\frac{\left|X_{i}-X_{i-1}\right|+\left|X_{i+1}-X_{i}\right|}{2}>$ spinthres,

where $X_{i+1}, X_{i}$ and $X_{i-1}$ represent three consecutive dBZ values along a radar radial, and "spinthres" is a reflectivity threshold. The number of sign changes is calculated within a window of 11 range gates around the centre range gate in a radial direction, as suggested by Hubbert et al. (2009). The reflectivity threshold is set to $5 \mathrm{dBZ}$ in Hubbert et al. (2009). Nevertheless, in several case studies, a threshold of $3 \mathrm{dBZ}$ turned out to perform best for the X-band radars used in this study. If both criteria are fulfilled in more than $10 \%$ of the consecutive range gates, the centre range gate is flagged as clutter.

Interferences caused by other radars occur in the form of spikes or rings in the radar image. Spikes are characterised and identified by a sign change in reflectivity difference between neighbouring radar beams, rings by a sign change in differences between neighbouring range gates. In order to identify spikes, the reflectivity difference between range gates of neighbouring radar beams is calculated and, from that, the sign change in differences is derived. If Eq. (3) is fulfilled in more than three cases within a window of five range gates in radial direction around the centre range gate, it is flagged as interference. The window of five range gates is chosen in order to be able to identify spikes that do not affect the entire radar beam. Equation (3) needs to be fulfilled for three instead of for all five range gates within the window to allow for detection of the edges of spikes, otherwise the first and last two range gates would remain in the radar image. To detect rings, the same procedure is applied by interchanging the radial and angular direction.

Despite the application of TDBZ, SPIN as well as spike and ring algorithms, some clutter remains in the radar image. The unique feature of high temporal resolution gives additional information for further clutter detection, in contrast to common radar systems: range gates with high reflectivities that are present in the current plan position indicator (PPI) image but not in the two previous ones are most likely clutter or interferences. In Fig. 5d-f, the PPI images of reflectivity fields without noise for 13:28:30 UTC (Fig. 5f) and the two previous time steps (13:27:30 UTC in Fig. 5d and 13:28 UTC in Fig. 5e) are shown. Red circles indicate examples of range gates that have reflectivity values greater than the noise level only at 13:28:30 UTC and are, therefore, identified as clutter. Clutter caused by moving objects as well as interferences due to external emitters are present for a short period of time. Therefore, it appears in only one time step, whereas precipitation structures remain nearly constant and between two time steps. For radars with a temporal resolution of the order of a few minutes, this method is not applicable.

Comparing the performance of the described dynamic clutter algorithms, the most efficient method is the TDBZ filter (Fig. 4b). It detects between 40 and $60 \%$ of all clutter, followed by the SPIN filter with 20 to $40 \%$. The algorithm for spike and ring identification can only detect interferences of other radars. These interferences are more rare than the static and dynamic clutter that is present in every radar image. Therefore, the spike and ring algorithms detect only between 15 and $35 \%$ of all clutter. The algorithm dependent on the advantage of high temporal resolution of the radar network comparing three time steps (PPI comp) is designed to identify both, clutter and interferences. It operates in rain-free areas and is, therefore, not directly comparable to the performance of the other three algorithms. Despite its limited applicability, it detects up to $10 \%$ of all clutter.

\subsubsection{Network-based clutter algorithm}

In contrast to single radar systems, networks can give multiple information on reflectivity in overlapping areas. For large-scale radar networks as operated by the national weather services, the areas of multiple coverage are minimised in order to cover an area as large as possible with a minimum number of radars. The X-band radar network in this study is designed so that a large area is covered by more than two radars. Each radar observes at a different height above a certain location. Obstacles in the near field of a radar will occur as clutter, e.g. the house in the image of radar A (Fig. 6a). The beam of radar B is higher above the ground at the same location and, therefore, is not affected. To ensure that a range gate is affected by clutter, the algorithm is 


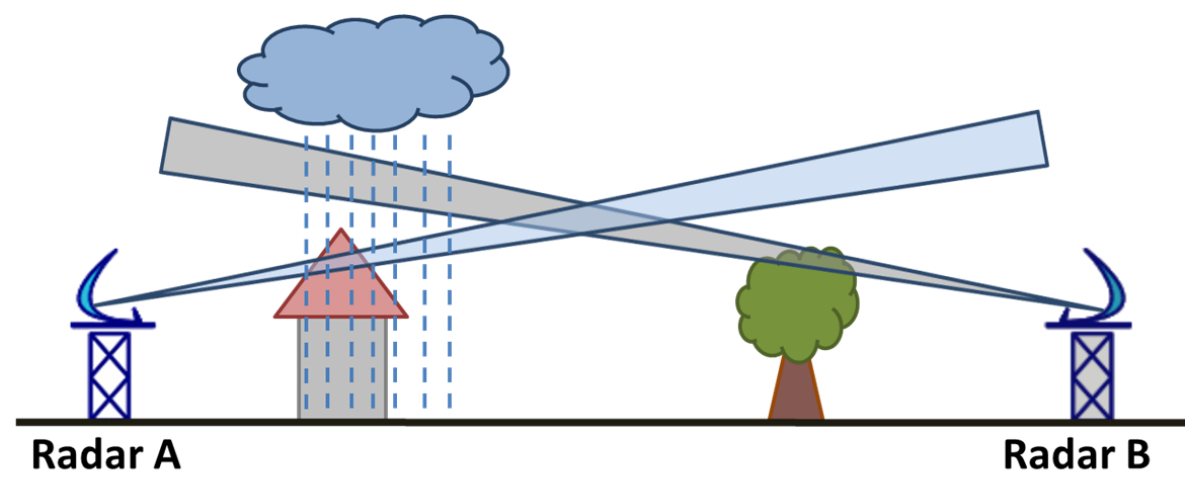

(a)

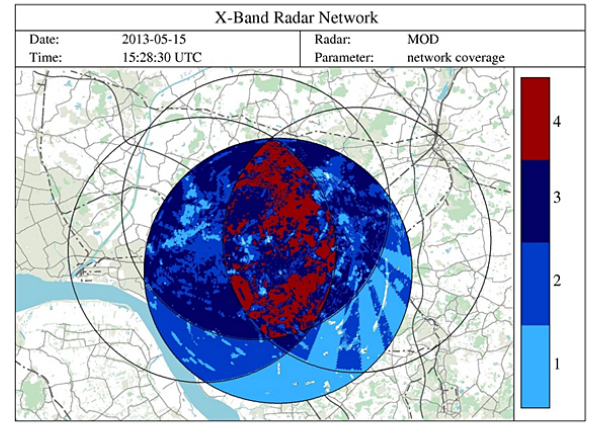

(b)

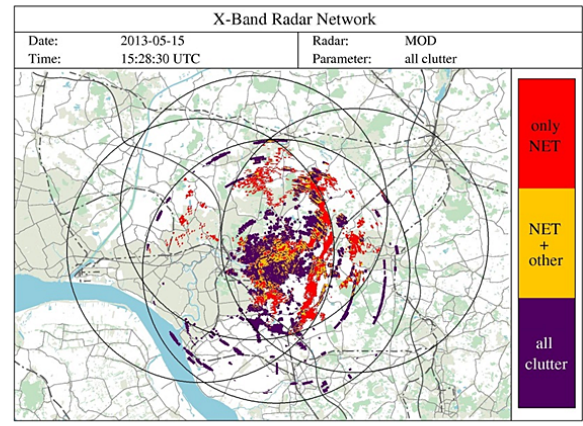

(c)

Figure 6. (a) Sketch of obstacles and precipitation as seen from two different radars; (b) coverage within the X-band radar network and (c) clutter range gates detected any clutter algorithm (violet) by the network algorithm and any other clutter algorithm (yellow), and by the network algorithm only (red).

applied in areas covered by at least three radars. If a range gate of one radar shows reflectivity, but the corresponding range gates of the other (at least two) radars do not, it is most likely to be clutter.

The areas covered by more than two radars for the example of 15 May 2013 at 15:28:30 UTC are indicated in dark blue and red in Fig. 6b. In this case that amounts to approximately $60 \%$ of the area covered by the radar. The area around the radar site is of special interest for clutter detection algorithms because most of the clutter occurs where the radar beam is close to the ground. This area is mostly covered by more than two radars and, therefore, the network-based clutter algorithm is applicable using the advantage of multiple coverage.

The added value of the network-based clutter algorithm is highlighted by the clutter map in Fig. 6c. The red area depicts all clutter range gates that are detected by no others than the network-based algorithm totals to approximately $30 \%$ of the clutter-affected area. In case of using a single radar only instead of a network, these disturbed range gates would remain in the radar image and cause erroneous precipitation estimates.

The long-term study of the performance of the networkbased clutter algorithm shows that this algorithm is the most efficient one (Fig. 4b). More than $60 \%$ and up to $80 \%$ of the clutter range gates are detected by the network algorithm. This is a very good performance, especially considering the fact that the network algorithm only works in areas that are covered by more than two radars.

\subsection{Calibration}

In order to calibrate reflectivity measurements of the X-band radar network, three reference stations are operated in the overlapping area of the PATTERN network. Each reference station consists of micro rain radar (MRR), a rain gauge and a wind sensor. The largest sources of error in rain gauge measurements are wind-induced losses. Thus, wind speed measurements from the wind sensor are used to correct $3 \mathrm{~h}$ averages of rain gauge measurements within the calibration period from April to October 2013 according to Rubel and Hantel (1999). These wind-corrected measurements from rain gauges are used to calibrate the micro rain radars. The MRRs at the reference stations derive rain-rate and reflectivity observations at 31 height levels from the ground to $1085 \mathrm{~m}$ in height. For measurements in the near field of the MRR, the relationship between power $P$ and distance $r$ is not valid, because the height resolution is almost of the order of the measuring height. The MRRs are operated with 


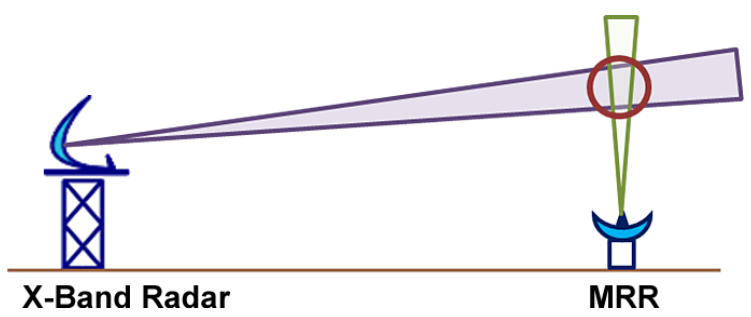

Figure 7. Sketch of the set-up for calibration of X-band radars with MRR.

a height resolution of $35 \mathrm{~m}$ and, therefore, it is common to omit the two lowest levels. The $P-r$ relation is applicable from the third level. MRR measurements are corrected for attenuation with the spectral scheme proposed by Peters et al. (2010) based on the classical attenuation correction scheme of Hitschfeld and Bordan (1954). This scheme avoids the uncertainty in the relationship between reflectivity $Z$ and rain rate $R$ by calculating attenuation $\kappa$ and $R$ from Doppler spectra using the drop size distribution. Three-hour averages of rain rate are calibrated with rain gauge measurements. The logarithmic calibration factor for MRR $\left(C_{\mathrm{MRR}}\right)$ rain rates $R$ and, therefore, also reflectivities, is the mean difference between the logarithmic rain rates of MRR ( $\left.\mathrm{dBR}_{\mathrm{MRR}}\right)$ and the rain gauge $\left(\mathrm{dBR}_{\mathrm{RG}}\right)$ :

$C_{\mathrm{MRR}}=\overline{\mathrm{dBR}_{\mathrm{MRR}}-\mathrm{dBR}_{\mathrm{RG}}}$ with $\mathrm{dBR}=10 \cdot \log (R)$.

The received signals of the MRR are transformed to drop size distributions (DSDs) using single-particle backscattering cross sections that are calculated with Mie theory using the code of Morrison and Cross (1974). Reflectivity $Z_{M R R}$ is derived from the MRR DSDs using Rayleigh approximation (Peters et al., 2005) and, thus, is independent of the wavelength. For the X-band radar, scattering is assumed to appear mainly as Rayleigh scattering, which is a good approximation for light and moderate rainfall. For high rain rates, it is difficult to separate the non-Rayleigh scattering effect from attenuation completely, due to rain. In this rain intensity range, attenuation by liquid water is of the same order or outweighs non-Rayleigh scattering effects. The good agreement between X- and C-band systems that is found by Barbieri et al. (2014) confirms the applicability of the Rayleigh approximation for X-band radars.

The X-band radars are calibrated with reflectivity measurements of MRRs. Therefore, directly measured reflectivity of the X-band radar is used for calibration, and not the precipitation product that is used for calibration with rain gauges. Another advantage of this method is that both systems observe reflectivity at the same height and, therefore, more or less in the same volume (Fig. 7). This volume is derived for each combination of X-band radar and MRR individually. The MRRs are between $1 \mathrm{~km}$ and $17.75 \mathrm{~km}$ away from the X-band radars. Depending on the distance to the radar, the radar beam is up to $870 \mathrm{~m}$ wide at the MRR sites.

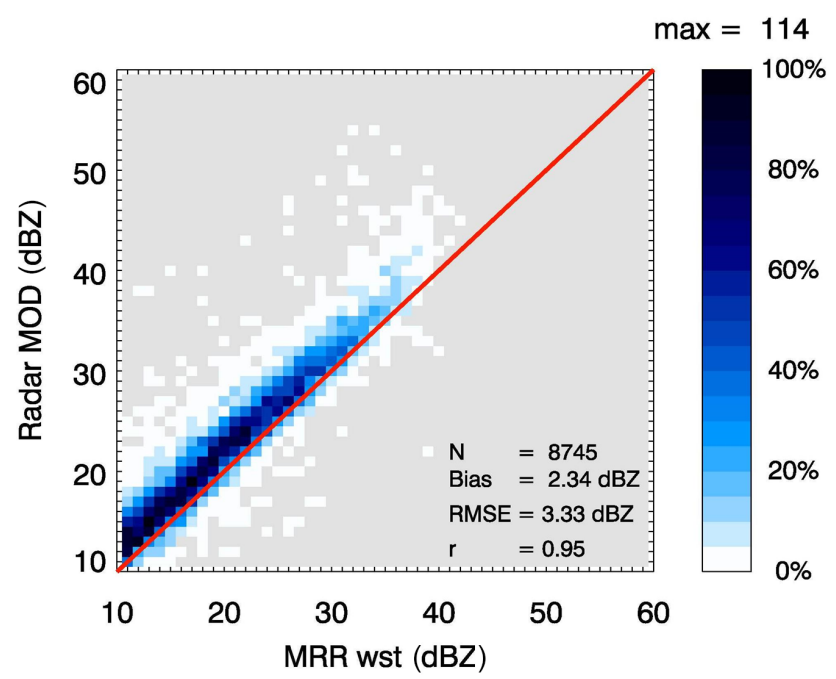

Figure 8. Comparison of X-band radar MOD (ordinate) to micro rain radar WST (abscissa) for April to October 2013. Frequency relative to the highest frequency is shown in different colours, from low levels in light blue to high levels in dark blue.

Therefore, up to $25 \mathrm{MRR}$ gates are within the radar beam. Reflectivities from all MRR gates that fall within the radar beam are averaged using a linear weighting function depending on their distance to the centre of the X-band radar beam, because the mean received power of the X-band radar comes from the centre of the beam. A linear fit of the form

$Z_{\mathrm{MRR}}=a \cdot Z_{X}$

is applied, where $Z_{\text {MRR }}$ is the reflectivity of the MRR, $Z_{X}$ the reflectivity of the X-band radar, and $a$ is the calibration coefficient:

$a=10^{0.1 \cdot\left(\mathrm{dBZ}_{X}-\mathrm{dBZ}_{\mathrm{MRR}}\right)}$.

As an example, a comparison between radar MOD and reference station WST is depicted in Fig. 8a for April to October 2013. Overall X-band radar MOD fits MRR WST measurements quite well, with a mean bias of $2.34 \mathrm{dBZ}$. The RMSE of $3.33 \mathrm{dBZ}$ is due to comparison on a $30 \mathrm{~s}$ basis. With a correlation coefficient of 0.95 , both systems are in very good agreement. A list of the calibration coefficients can be found in Table 2 .

\subsection{Attenuation correction}

As the X-band frequency range is highly influenced by attenuation, parts of an algorithm especially developed for small single-polarised X-band radars are used according to Delrieu et al. (1997), based on Marzoug and Amayenc (1994). Attenuation $A(r)$ at a range gate $r$ is multiplicative, and the true reflectivity profile $Z(r)$ can be calculated with the measured reflectivity profile $Z_{\mathrm{m}}(r)$ and a constant radar calibrationerror 
Table 2. Calibration coefficients $a$ for every possible combination of X-band radar (HWT, MOD, QNS and BKM) and MRR (OST, MST and WST) and the average coefficient in $\mathrm{dBZ}$.

\begin{tabular}{lllll}
\hline & $a_{\text {OST }}$ & $a_{\text {MST }}$ & $a_{\text {WST }}$ & $a$ \\
\hline HWT & 0.74 & 1.00 & 0.91 & 0.88 \\
MOD & 2.31 & 3.63 & 2.34 & 2.76 \\
QNS & 5.67 & 5.63 & - & 5.65 \\
BKM & 0.75 & 0.90 & - & 0.83 \\
\hline
\end{tabular}

$\delta C$ (see Table 1):

$Z_{\mathrm{m}}(r)=Z(r) \delta C A(r)$

Attenuation influences the radar beam in both ways, away from and back to the radar. Therefore, two-way apparent attenuation $K$ is calculated as an integral along the path from range gate 0 to range gate $r$ and back:

$K(r)=2 \int_{0}^{r}\left[\frac{Z_{\mathrm{m}}(s)}{\alpha}\right]^{\frac{1}{\beta}} \mathrm{d} s$,

with coefficients $\alpha=132250$ and $\beta=1.2$ for the X-band frequency range (Delrieu et al., 1997). The attenuation factor $A$ for a certain range gate $r$ is then calculated as

$A(r)=\left[1-\frac{0.23}{\beta} K_{\mathrm{m}}(r)\right]^{\beta}$.

The determination of attenuation works along a path with undisturbed measurements. Because of the elimination of clutter-affected range gates, data gaps occur that need to be filled. A common method for single radars is interpolation of data gaps. Thereby, information on the small-scale variability of the rain event gets lost. In the overlapping areas within the network, the advantages of multiple information from different radars are used to fill data gaps. Therefore, the structure of precipitation is kept, and allows for more precise attenuation estimation within the network compared to single radars. The attenuation factor field is presented in Fig. 9a. It is in good agreement with the finding in Doviak and Zrnic (1993). In areas of high reflectivities in the most southern and western parts of the radar image, the attenuation factor $A(r)$ is higher than 0.5 . The corrected reflectivity field after clutter filtering, calibration and attenuation correction is shown in Fig. $9 b$.

\subsection{Composite of network radars}

The X-band radar network derives multiple information on reflectivity, clutter and attenuation, because a large area is covered by more than one radar. To combine information of all four radars, a composite is calculated on a rotated Cartesian grid; i.e., the Equator is shifted into the network-covered area to allow for equidistant grid cells. A grid resolution of $250 \mathrm{~m}$ is used. Each radar range gate is assigned to the grid cell its centre is located in and the average of reflectivity values of all radar range gates in a certain grid cell is calculated. In the outer parts of the radar-covered area, radar range gates are much larger in the azimuthal direction than grid cells and, therefore, not every grid cell includes a radar range gate centre. To make sure that grid boxes far away from the radar sites contain at least one radar range gate centre, the resolution of the radar is artificially enhanced by dividing each azimuth angle into $0.1^{\circ}$ steps. In order to determine rainfall rates $R$ of the composite of reflectivities (Fig. 10), a common Z-R relation is applied:

$R=a Z^{b}$,

with coefficients $a=320,200$ or 77 and $b=1.4,1.6$ or 1.9, respectively, depending on the strength of the rain event. These coefficients are used for precipitation estimation in Germany by the DWD, and are adopted for the X-band radar measurements in order to allow for comparison with precipitation rates obtained from the C-band radar operated by the DWD. The result is a nearly undisturbed precipitation field that covers the western half of the network area.

\section{Comparison to C-band radar}

In the last section, it was shown that several algorithms are needed to obtain nearly undisturbed calibrated reflectivity fields from raw data. In order to give an estimation on the quality of products from the PATTERN X-band radar network, reflectivity data are compared to the products of radar Fuhlsbüttel operated by the DWD in Hamburg $40 \mathrm{~km}$ southeast of the network area. Radar Fuhlsbüttel provides reflectivity measurements in the C-band frequency range, with a range resolution of $1 \mathrm{~km}$, an azimuthal resolution of $1^{\circ}$ and a temporal resolution of $5 \mathrm{~min}$. In this study, the precipitation scan with an elevation angle of $0.7^{\circ}$ is used for comparison to $\mathrm{X}$-band radar data. The PATTERN network observes reflectivity with a temporal resolution of $30 \mathrm{~s}$. Therefore, reflectivity fields obtained by the PATTERN network are compared to the closest 5 min measurements of radar Fuhlsbüttel.

A comparison of the composite of reflectivity fields of the four PATTERN radars (Fig. 11a) for 15 May 2013, 15:28:30 UTC to the product of radar Fuhlsbüttel (Fig. 11b) for 15 May 2013, 15:30 UTC indicates the high spatial accordance of both systems. Both, geographical position of the precipitation area as well as its maxima in the western part of the network area, are displayed well by the PATTERN radars and radar Fuhlsbüttel. Nevertheless, reflectivity values are slightly higher in the PATTERN network composite than for radar Fuhlsbüttel. This is due to the different resolutions of both systems. Maximum reflectivities observed by the highresolution X-band radar are smoothed by the $\mathrm{C}$-band radar. Another possible explanation for the relative bias might be 


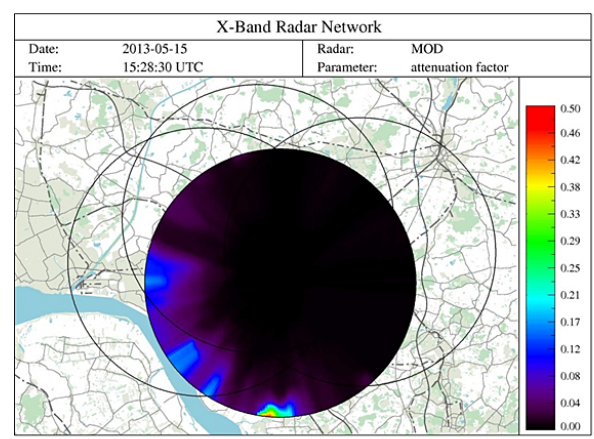

(a)

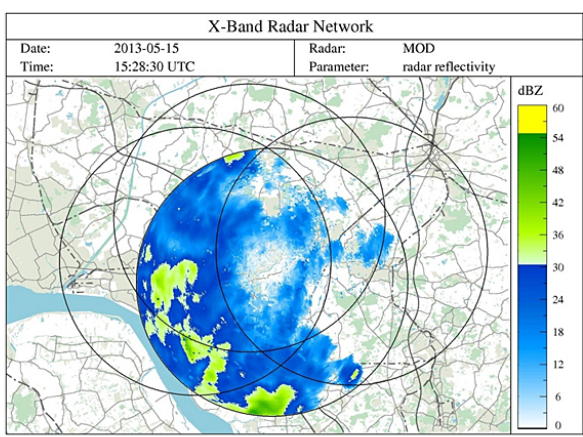

(b)

Figure 9. Attenuation correction for radar MOD for 15 May 2013, 15:28:30 UTC: (a) attenuation factor field and (b) corrected dBZ field.

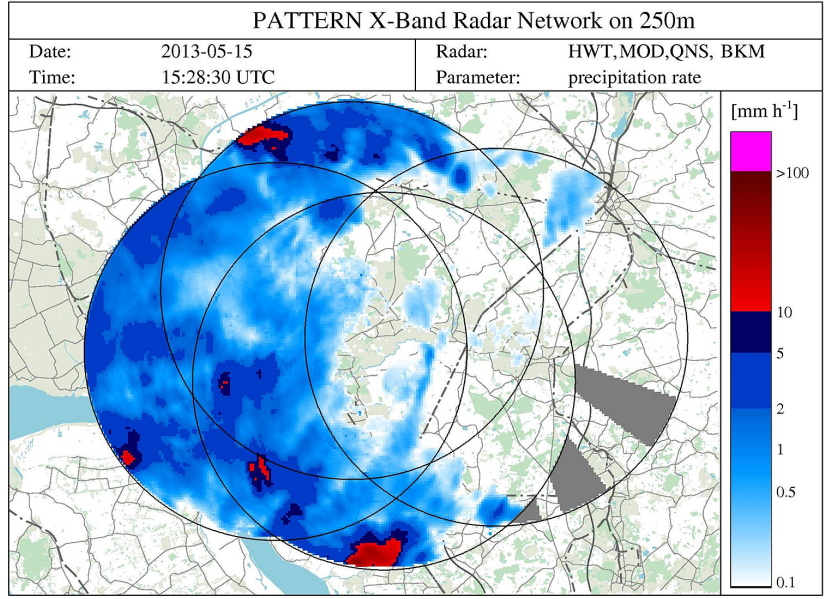

Figure 10. Composite of the precipitation fields of all four PATTERN radars for 15 May 2013, 15:28:30 UTC.

the different calibrations of both systems. The precipitation field is slightly shifted to the east in the image derived by radar Fuhlsbüttel. This is due to the time shift of $1.5 \mathrm{~min}$ between Fig. 11a and b.

The good agreement between the PATTERN network and radar Fuhlsbüttel in terms of reflectivity is also evident in a long-term comparison of both systems shown in Fig. 12. Reflectivities from the PATTERN network are averaged on the grid of radar Fuhlsbüttel. All precipitation events that occur from May to September 2013 are taken into account, and reflectivity values are divided into $1 \mathrm{dBZ}$ steps. Overall, both systems are in good agreement. The PATTERN network slightly overestimates measurements of radar Fuhlsbüttel for reflectivities lower than $15 \mathrm{dBZ}$, which results in an intercept of $6.7 \mathrm{dBZ}$ when DWD radar is used as a regressor. For PATTERN radar as a regressor, the intercept is clearly smaller, at $1.5 \mathrm{dBZ}$. The probability of detection (POD) and the false alarm ratio (FAR) give additional information on the accordance of both systems. One system serves as a reference, and the other system's ability to observe the same precipitation events is tested. POD is a measure of how many of all precipitation events detected by the reference system are also observed by the test system. It is $90 \%$ for LAWR as a test system, and $93 \%$ for DWD C-band radar as a test system. This means that at least $90 \%$ of all rain events are measured by both systems. POD is slightly smaller for the $\mathrm{X}$-band radar because small-scale structures, especially at the border of rain events, cannot be resolved by the C-band radar. FAR is a measure of how often the test system detects rainfall, while the reference does not observe any precipitation. It does not exceed $10 \%$ for either system. The good agreement in terms of POD, FAR and reflectivity values between PATTERN and radar Fuhlsbüttel demonstrates that, overall, the PATTERN network provides reliable reflectivity data and promising results in terms of higher resolution.

The higher resolution of the PATTERN product compared to radar Fuhlsbüttel allows for enhanced and more detailed spatial allocation of precipitation. In order to investigate the variability of reflectivity within a single range gate of radar Fuhlsbüttel with a range resolution of $1 \mathrm{~km}$ and an azimuthal resolution of $1^{\circ}$, the number of rain range gates from the PATTERN network is calculated for each DWD range gate (Fig. 13a). The lower the percentage of rain range gates, the smaller the rain-covered area within a certain DWD range gate is. The western part of the network is completely covered by rain and, therefore, the percentage of rain range gates from the network is $100 \%$ for almost all DWD range gates. At the edges of the precipitation field in the centre of the network area, the percentage drops to less then $10 \%$. These small-scale structures cannot be observed with the coarse resolution of radar Fuhlsbüttel. The standard deviation within each DWD range gate, depicted in Fig. 13b, stresses the importance of high-resolution precipitation observations, with values of up to $3 \mathrm{dBZ}$ at the edges of the rain events.

\section{Conclusions}

A network consisting of four X-band radars has been deployed within the framework of the Precipitation and 


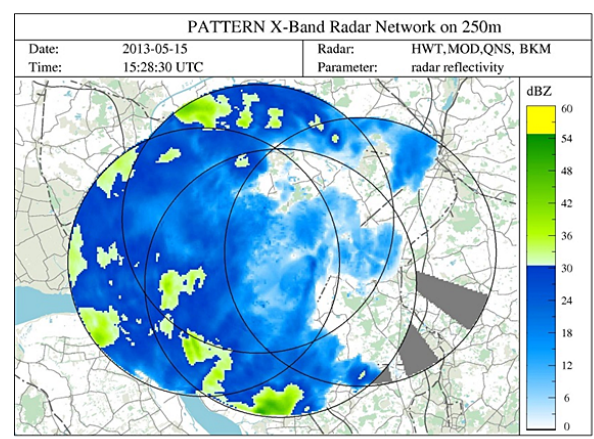

(a)

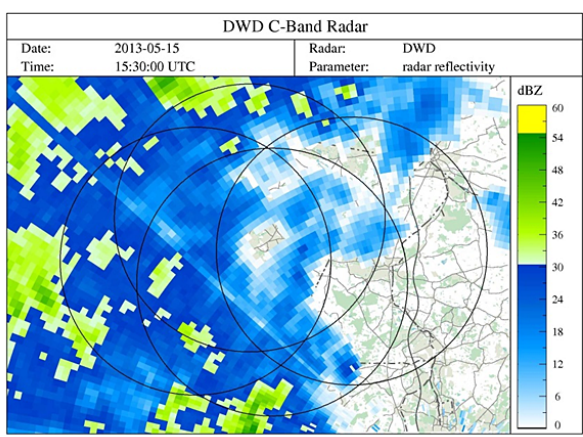

(b)

Figure 11. (a) Composite of reflectivity fields of all four network radars for 15 May 2013, 15:28:30 UTC, and (b) reflectivity field from radar Hamburg for 15 May 2013, 15:30 UTC.

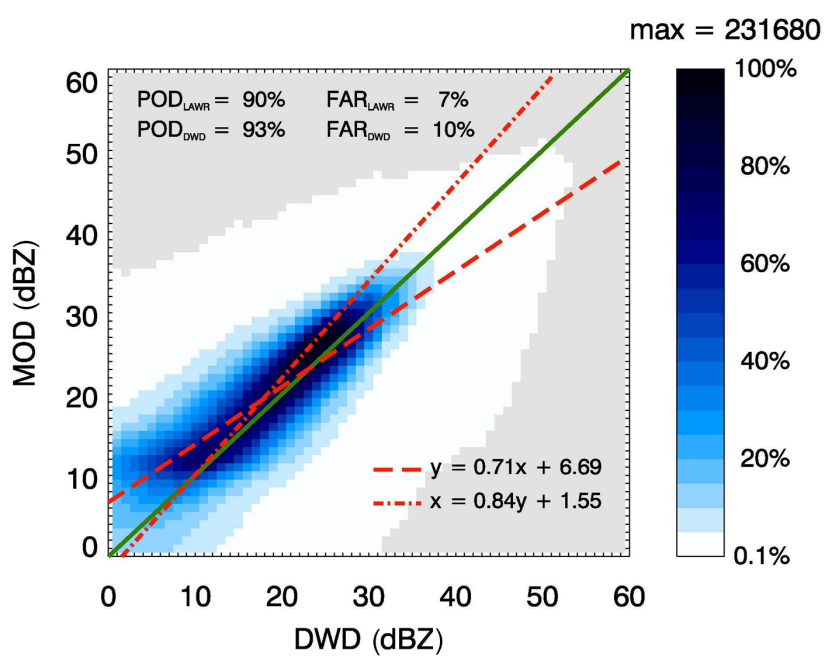

Figure 12. Comparison of reflectivity values from radar Fuhlsbüttel (abscissa) and radar MOD (ordinate) from May to September 2013. Frequency relative to the highest frequency is shown in different colours, from low levels in light blue to high levels in dark blue. Dashed and dashed-dotted red lines denote linear fits with radar Fuhlsbüttel and radar MOD as regressors, respectively. The probability of detection (POD) and the false alarm ratio (FAR) are also given.

Attenuation Estimates from a High-Resolution Weather Radar Network (PATTERN) project that has been operational since January 2012. The radars provide reflectivity fields with a range resolution of $60 \mathrm{~m}$, a sampling resolution of $1^{\circ}$ in the azimuthal direction, and a temporal resolution of $30 \mathrm{~s}$. Algorithms have been developed to remove disturbances in raw reflectivity fields of single radars. The performance of these algorithms is exemplary, as shown for one of the network radars (MOD). The simple radar systems presented in this study cannot measure background noise directly. Therefore, noise level is estimated for each radar using the 5th percentile of the smoothed reflectivity field and the noise levels of the last 10 time steps.
Approximately $10 \%$ of the radar-covered area is disturbed by reflection from obstacles such as trees or houses or from other transmitters. In order to identify clutter range gates, two different types of clutter detection algorithms are applied: single-radar and network-based algorithms. Some of the single-radar algorithms are based on classic clutter detection methods, such as the texture of the dBZ field (TDBZ), sign changes in reflectivity gradient between neighbouring range gates (SPIN), the shape of disturbances from other transmitters (spikes or rings), or a static clutter map. The most efficient of these single-radar algorithms is the TDBZ filter, which detects up to $60 \%$ of all clutter, followed by the SPIN filter with up to $40 \%$.

The X-band radars do not perform dual-polarisation measurements, but the network of X-band radars introduced in this paper has two other features that are beneficial for clutter detection: high temporal resolution and multiple coverage by more than two radars within the network area. The advantage of a high temporal resolution of $30 \mathrm{~s}$ is used for clutter detection by comparing the PPI image of the current time step to the two previous ones. Range gates with reflectivity values higher than the noise level that do not occur in the two previous images are flagged as clutter. This type of algorithm only works for high temporal resolution, because the spatial shift in the precipitation field is small compared to a time step of $5 \mathrm{~min}$, which is the common temporal resolution of operational regional radar systems. Around $10 \%$ of clutter can be detected with this type of algorithm that operates in rain-free areas. The second type of clutter algorithm is based on the advantage of having a network of four radars with high spatial resolution. In areas covered by more than two radars, range gates with reflectivities higher than the noise level that do not appear in at least two other radars are flagged as clutter. This network-based clutter algorithm is more efficient than all single radar algorithms, with a detection rate of more than $70 \%$. Therefore, the network of X-band radars is a very useful tool for clutter filtering that is also used to fill the gaps resulting from clutter filtering. Thus, smoothing of the reflectivity field 


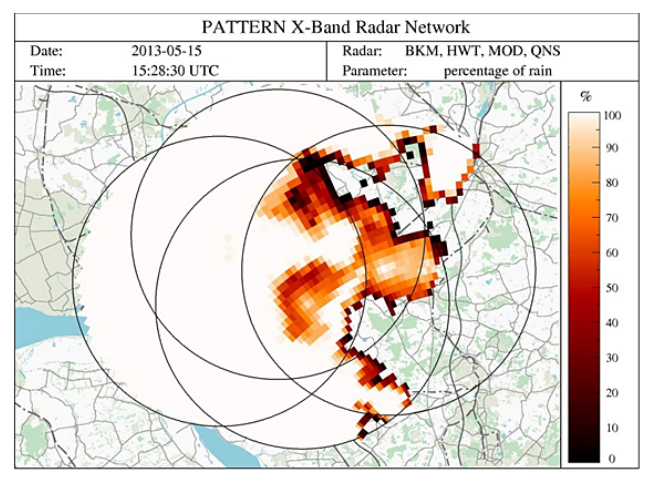

(a)

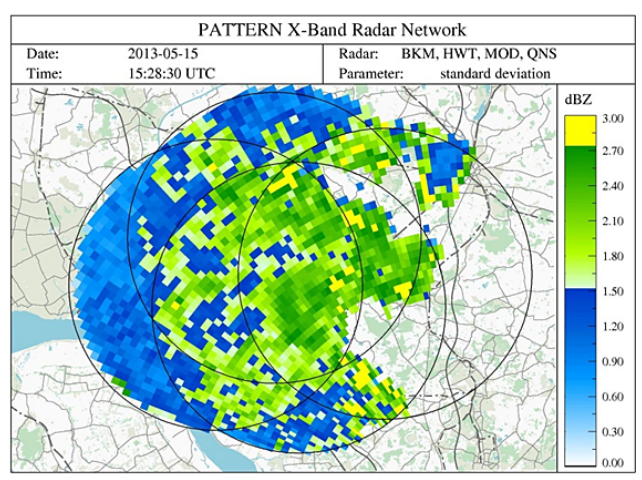

(b)

Figure 13. (a) Percentage of PATTERN network range gates with rain on the DWD grid and (b) standard deviation of reflectivity in the PATTERN network on the DWD grid for 15 May 2013, 15:28:30 UTC.

due to interpolation is avoided, and the small-scale structure of rain events is kept.

Three reference stations are deployed within the networkcovered area for calibration purposes, consisting of a rain gauge, a wind sensor, and micro rain radar (MRR). Using MRRs for X-band radar calibration has the advantage of comparing reflectivity measurements at the same height level instead of to observations at the ground from rain gauges. A slight overestimation of MRR measurements is detected and corrected for radar MOD. X-band radars are highly influenced by attenuation from liquid water. Therefore, a simple single-radar algorithm for attenuation correction is applied to the network radars. In order to apply this algorithm, a continuous reflectivity field without data gaps caused by clutter and interferences is needed. Here, the network benefits again from the large area covered by more than one radar. Information from other radars is used to fill these gaps and maintain the small-scale structure of rain events instead of smoothing the precipitation field by interpolation.

A composite of all four radars is calculated on a $250 \mathrm{~m} \times 250 \mathrm{~m}$ grid by averaging reflectivities from all range gates whose centres fall within a certain grid cell. A comparison to measurements from a C-band radar operated by DWD indicates that the PATTERN network slightly overestimates reflectivity but also displays the spatial structure of rain events very well in higher resolution than nationwide radar networks can do. This is in good agreement with case studies conducted by Allegretti et al. (2012) and Trabal et al. (2013), who recommend the use of low-cost X-band radars in complementation to large-scale C- or S-band networks.

A long-term study showed that both systems are in good agreement for all rain events that occurred from May to September 2013. It has been shown that, especially at the border of rain events, where only parts of the C-band radar range gates are covered by rain, higher resolution of the $\mathrm{X}$-band radar network provides more detailed information on the structure of the precipitation. Within a C-band radar range gate, the standard deviation can be up to $3 \mathrm{dBZ}$. Due to its low costs compared to other radar systems (less than $20 \%$ of the price of dual-polarisation systems), a single LAWR or a network of LAWRs is affordable not only for weather services, but also for private companies and local authorities, and can be set up in areas of special interest, e.g. urban areas or mountainous regions. They can serve as a sort of magnifying glass to investigate the spatial and temporal structures of rain events in addition to large S- or C-band radar systems.

\section{Outlook}

It has been shown in this paper that a network of LAWRs gives reliable precipitation estimates and can be a useful addition to nationwide radar networks. For further improvement, the next step will be the implementation of attenuation correction algorithms that use the advantage of a network (e.g. Chandrasekar and Lim, 2008; Srivastava and Tian, 1996; Testud and Amayenc, 1989). Better estimation of attenuation can lead to better precipitation estimates, because the relation between attenuation and precipitation is more stable than the relation between reflectivity and precipitation.

Furthermore, the fixed relation between radar reflectivity and precipitation will be replaced by a dynamic relation determined operationally using measurements of seven MRRs installed in the PATTERN catchment. This allows for adaptation of $\mathrm{Z}-\mathrm{R}$ relations to current weather conditions, e.g. showers and light or stratiform rain.

High-resolution products of the PATTERN network will also be used as input for rainfall-runoff simulations. Currently, hydrometeorological models use products from C- or S-band radars as input, with a resolution of several minutes in time and kilometres in space. Higher spatial and temporal resolution of precipitation estimates can be used to improve rainfall-runoff simulations in areas of special interest, e.g. in small-scale structured urban areas. 
Acknowledgements. The Precipitation and Attenuation Estimates from a High-Resolution Weather Radar Network (PATTERN) project is a joint project between the University of Hamburg and the Max-Planck-Institute for Meteorology. It is funded by the Deutsche Forschungsgemeinschaft (grant AM308/3-1).

The authors thank the German Weather Service (DWD) for making products of their C-band radar network available for research purposes within the PATTERN project.

Edited by: G. Vulpiani

\section{References}

Allegretti, M., Bertoldo, S., Prato, A., Lucianaz, C., Rorato, O., Notarpietro, R. and Gabella, M.: X-Band Mini Radar for Observing and Monitoring Rainfall Events, Atmos. Clim. Sci., 2, 290-297, 2012

Atlas, D. and Banks, H. C.: The Interpretation of Microwave Reflections from Rainfall, J. Meteor., 8, 271-282, 1951

Atlas, D. and Ulbrich, C. W.: Path- and Area-Integrated Rainfall Measurement by Microwave Attenuation in the 1-3 cm Band, J. Appl. Meteor., 1, 1322-1331, 1977

Barbieri, S., Piciotti, E., Montopoli, M., Di Fabio, S., Lidori, R., Marzano, F., Kalogiros, J., Anagnostou, M., and Baldini, L.: Intercomparison of Dual-Polarization X-Band Mini-Radar Performances with Reference Radar Systems at X- and C-Band in Rome Supersite, Proceedings of 8th European Conference on Radar in Meteorology and Hydrology, Garmisch-Partenkirchen, Germany, 1-5 September 2014

Beck, J. and Bousquet, O.: Using Gap-Filling Radars in Mountainous Regions to Complement a National Radar Network: Improvements in Multiple-Doppler Wind Syntheses, J. Appl. Meteor., 52, 1836-1850, 2013

Chandrasekar, V. and Lim, S.: Retrieval of Reflectivity in a Networked Radar Environment, J. Atmos. Oceanic Technol., 25, 1755-1767, 2008

Delrieu, G., Caoudal, S., and Creutin, J. D.: Feasibility of Using Mountain Return for the Correction of Ground Based X-Band Weather Radar, J. Atmos. Ocean. Technol., 14, 368-385, 1997

Doviak, R. J. and Zrnic, D. S.: Doppler Radar and Weather Observations, Dover Publications, 2nd Edn., 42, 1993

Dutton, E. J.: Estimation of Radio Attenuation in Convective Rainfalls, J. Appl. Meteor., 6, 622-668, 1967

Einfalt, T.: A User Perspective in Germany: What is Espected by Agencies and Government from Radar Data?, Int. J. River Basin Management, 1, 1-5, 2003

Gunn, K. L. S. and East, T.: The Microwave Properties of Precipitation Particles, Quart. J. R. Meteor. Soc, 80, 522-545, 1954

Hitschfeld, W. and Bordan, J.: Errors Inherent in the Radar Measurement of Rainfall at Attenuating Wavelengths, J. Meteorol., 11, 58-67, 1954.

Hubbert, J. C., Dixon, M., and Ellis, S. M.: Weather Radar Ground Clutter. Part II: Real-Time Identification and Filtering, J. Atmos. Ocean. Technol., 26, 1181-1197, 2009

Kabeche, A. C. and Testud, J.: Stereoradar Meteorology: A New Unified Approach to Process Data from Airborne or GroundBased Meteorological Radars., J. Atmos. Ocean. Technol., 12, 783-799, 1995
Lengfeld, K., Clemens, M., Feiertag, N., and Ament, F.: Precipitation and Attenuation Estimates from a High-Resolution Weather Radar Network (PATTERN): Design of the Experiment, in: Proceedings of the 7th European Conference on Radar in Meteorology and Hydrology, Toulouse, France, 24-29 June 2012

Lengfeld, K., Clemens, M., Münster, H., and Ament, F.: PATTERN: Advantages of High-Resolution Weather Radar Networks, in: Proceedings of American Meteorological Society 36th Conference on Radar Meteorology, Breckenridge, CO, USA, 16-20 September 2013.

Lim, S., Chandrasekar, V., Lee, P., and Jayasumana, A. P.: RealTime Implementation of a Network-Based Attenuation Correction in the CASA IP1 Testbed, J. Atmos. Ocean. Technol., 28, 197-209, 2011

Mahale, V. N., Brotzge, J. A., and Bluestein, H. B.: The Advantages of a Mixed-Band Radar Network for Severe Weather Operations: A Case Study of 13 May 2009, Weather Forecast., 29, 78-98, 2014

Marzoug, M. and Amayenc, P.: A Class of Single- and DualFrequency Algorithms for Rain-Rate Profiling from a Spaceborne Radar. Part I: Principle and Tests from Numerical Simulations, J. Atmos. Ocean. Technol., 11, 1480-1506, 1994

Matrosov, S. Y., Cifelli, R., and Gochis, D.: Measurements of Heavy Convective Rainfall in the Presence of Hail in Flood-Prone Areas Using an X-Band Polarimetric Radar, J. Appl. Meteorol., 52, 395-407, 2013

McLaughlin, D., Pepyne, D., Chandrasekar, V., Philips, B., Kurose, J., Zink, M., Droegemeier, K., Cruz-Pol, S., Junyent, F., Brotzge, J., Westbrook, D., Bharadwaj, N., Wang, Y., Lyons, E., Hondl, K., Liu, Y., Knapp, E., Xue, M., Hopf, A., Kloesel, K., DeFonzo, A., Kollias, P., Brewster, K., Contreras, R., Dolan, B., Djaferis, T., Insanic, E., Frasier, S. and Carr, F.: Short-Wavelength Technology and the Potential for Distributed Networks of Small Radar Systems, B. Am. Meteor. Soc., 90, 1797-1817, 2009

Morrison, J. A. and Cross, M. J.: Scattering of a Plane Electromagnetic Wave by Axissymmetric Raindrops, Bell. Syst. Tech. J., 53, 955-1019, 1974

Peters, G., Fischer, B., and Clemens, M.: Rain Attenuation of Radar Echeos Considering Finite-Range Resolution and Using Drop Size Distributions, J. Atmos. Ocean. Technol., 27, 829-842, 2010

Peters, G., Fischer, B., Münster, H., Clemens, M. and Wagner A.: Profiles of Raindrop Size Distributions as Retrieved by Micro Rain Radars, J. Appl. Meteorol., 44, 1930-1949, 2005

Rubel, F. and Hantel, M.: Correction of Daily Rain Gauge Measurements in the Baltic Sea Drainage Basin, Nord. Hydrol., 30, 191-208, 1999

Shakti, P. C., Maki, M., Shimizu, S., Maesaka, T., Kim, D.-S., Lee, D. I., and Iida, H.: Correction of Reflectivity in the Presence of Partial Beam Blockage over a Mountainous Region Using XBand Dual Polarization Radar, J. Hydrometeorol., 14, 744-764, 2013

Skolnik, M. I. (Ed.): Radar Handbook, McGraw-Hill, New York, 3rd Ed, 2008.

Srivastava, R. C. and Tian, L.: Measurements of Attenuation by a Dual-Radar Method, J. Atmos. Ocean. Technol., 13, 937-947, 1996 
Tian, L. and Srivastava, R. C.: Measurements of Attenuation at CBand in a Convective Storm by a Dual-Radar Method, J. Atmos. Ocean. Technol., 14, 184-196, 1997

Testud, J. and Amayenc, P.: Stereoradar Meteorology: A Promising Technique for Observation of Precipitation from a Mobile Platform, J. Atmos. Ocean. Technol., 6, 89-108, 1989

Trabal, J. M., Colom-Ustariz, J., Cruz-Pol, S., Pablos-Vega, G. A., and McLaughlin, D. J.: Remote Sensing of Weather Hazards Using a Low-Cost and Minimal Infrastructure Off-the-Grid Weather Radar Network, IEEE Trans. Geosci. Remote Sens., 51, 25412555, 2013
Turso, S., Zambotto, M., Gabella, M., Orione, F., Notarpietro, R., and Perona, G.: Microradarnet: An Innovative Wireless Micro Radar Network for High Resolution Weather Monitoring, presented at 9th European Conference on Applications of Meteorology, Toulouse, France, 28 September-2 Oktober 2009, 2009

Ventura, J. F. I. and Tabary, P.: The New French Operational Polarimetric Radar Rainfall Rate Product, J. Appl. Meteor., 52, 18171835,2013

Wexler, R. and Atlas, D.: Radar Reflectivity and Attenuation of Rain, J. Appl. Meteor., 2, 276-280, 1963 